\title{
Cytotoxic lesions of the corpus callosum (CLOCCs) associated with SARS-CoV-2 infection
}

\author{
Anthony Moreau ${ }^{1}$ (1) $\cdot$ Amedee Ego $^{1} \cdot$ Frederic Vandergheynst $^{2} \cdot$ Fabio Silvio Taccone $^{1} \cdot$ Niloufar Sadeghi $^{3}$. \\ Isabel Montesinos ${ }^{4} \cdot$ Nicolas Gaspard ${ }^{5,6}$. Julie Gorham ${ }^{1}$
}

Received: 27 May 2020 / Revised: 9 August 2020 / Accepted: 12 August 2020 / Published online: 18 August 2020

○) Springer-Verlag GmbH Germany, part of Springer Nature 2020

\section{Dear Sirs,}

Although coronavirus disease-2019 (COVID-19) mostly affects the respiratory system [1], neurological manifestations have already been described in COVID-19 patients [2] Here, we report a case of neurological symptoms associated with cytotoxic lesions of the corpus callosum (CLOCCs) in a COVID-19 patient.

A 26-year-old patient, without any medical history, was admitted for dry cough and acute confusion for 2 days. He was awake but agitated and disoriented, exhibiting violent behavior and inappropriate speech. On clinical examination, no focal or meningeal signs were reported. Body temperature was $40{ }^{\circ} \mathrm{C}$ and oxygen saturation on room air was $100 \%$. Laboratory tests results included increased C-reactive protein level [200 mg/l; normal value (NV): $<5 \mathrm{mg} / \mathrm{l}]$, lym-

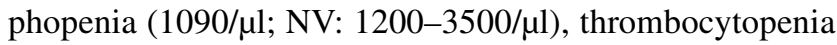
$\left(95 \times 10^{3} / \mu \mathrm{l}\right.$; NV: $\left.150-440 \times 10^{3} / \mu \mathrm{l}\right)$, elevated D-dimers (4466 ng/ml; NV: $<500 \mathrm{ng} / \mathrm{ml})$, ferritin $(1278 \mu \mathrm{g} / \mathrm{l}$; NV: 30-300 $\mu \mathrm{g} / \mathrm{l})$, high-sensitivity troponin I (226 ng/l;

Anthony Moreau

Anthony.Moreau@ulb.be

1 Department of Intensive Care, Erasme Hospital, Université Libre de Bruxelles (ULB), Route de Lennik, 808, 1070 Brussels, Belgium

2 Department of Internal Medicine, Erasme Hospital, Université Libre de Bruxelles (ULB), Brussels, Belgium

3 Department of Neuroradiology, Erasme Hospital, Université Libre de Bruxelles (ULB), Brussels, Belgium

4 Microbiology Department, Laboratoire Hospitalier Universitaire de Bruxelles-Universitair Laboratorium Brussel (LHUB-ULB), Université Libre de Bruxelles, Brussels, Belgium

5 Department of Neurology, Erasme Hospital, Université Libre de Bruxelles (ULB), Brussels, Belgium

6 Department of Neurology, Yale University, New Haven, CT, USA
$\mathrm{NV}<14 \mathrm{ng} / \mathrm{l})$ and IL-6 (132 pg/ml; NV $\leq 1.8 \mathrm{pg} / \mathrm{ml})$ levels. Cerebro-spinal fluid was crystal clear, with $3 / \mu \mathrm{l}$ white blood cell and normal protein levels. Polymerase chain reaction (PCR) on CSF for neurotropic viruses was negative. Search for autoantibodies and serological tests for human immunodeficiency virus and syphilis tested negative. Three reverse real-time PCR (rRT-PCR) assays for SARS-CoV-2 on nasopharyngeal swabs were negative. Trans-thoracic echocardiography revealed biventricular dysfunction with diffuse hypokinesia and left ventricular ejection fraction of 35\%, suggestive of myocarditis. Thin-section chest computed tomography showed bilateral crazy-paving patterns, suggestive of COVID-19. Brain magnetic resonance imaging (MRI; Fig. 1) on admission revealed a round lesion in the splenium of the corpus callosum, hyperintense on fluid attenuated inversion recovery (FLAIR) and T2-weighted imaging with restricted diffusion, compatible with the diagnosis of cytotoxic lesions of the corpus callosum (CLOCCs). An electroencephalogram showed mild encephalopathy with no epileptiform discharges. Serological blood test for SARS-CoV-2 IgG (DiaSorin-Saluggia, Italy) tested positive (34 AU/ml; cutoff $=12 \mathrm{AU} / \mathrm{ml})$. The treatment was conservative. The neurological status of the patient improved in the next $48 \mathrm{~h}$ and his cardiac dysfunction resolved within a week. Control MRI performed three weeks later was normal (Fig. 1).

CLOCCs are non-specific findings on brain MRI associated with reversible neurological signs, such as behavior changes and multiple etiologies, including viral illness, drug toxicity, seizures, malignancy, subarachnoid hemorrhage and metabolic disturbances [3]. The physiopathological hypothesis is that an inflammatory process involving cytokines such as IL-6 triggers the accumulation of glutamate in the extracellular space, resulting in cytotoxic edema, in particular of astrocytes. The selective vulnerability of the corpus callosum could be explained by its high density of cytokine and glutamate receptors [3]. CLOCCs has been reported in several viral infections [3], but this is the first description 
Fig. 1 Brain magnetic resonance imaging (MRI), performed the day after admission, revealed the presence of a well-circumscribed oval lesion within the splenium of the corpus callosum. The lesion demonstrates the features of cytotoxic edema: hyperintensity in fluid-attenuation inversion recovery (FLAIR) sequence (a) and restricted diffusion displayed by an area of high signal intensity on diffusionweighted imaging (DWI; b) and low signal intensity on apparent diffusion coefficient (ADC; c) map. There is no hemorrhage on gradient echo T2-weighted imaging (d). Control MRI showed complete resolution of the lesion on FLAIR (e) and DWI (f) sequences



of association with SARS-CoV-2 infection. Clinical and laboratory findings were not suggestive of any of the other above-mentioned conditions. Despite two negative rRT-PCR assays, chest-CT findings, high IL-6 level [4] and a positive serological test confirmed the diagnosis of COVID-19, which had likely occurred at least 2 weeks before admission [5]. This case further expands the spectrum of acute neurological complications of COVID-19. Mao et al. [2] reported that $36 \%$ of COVID-19 patients had neurological manifestations, including encephalopathy, acute cerebrovascular diseases and skeletal muscle injury. Interestingly, complications such as Guillain-Barré syndrome, Miller-Fisher syndrome or demyelinating lesions of the central nervous system [6-8] have also been reported, suggesting immunemediated post-infectious mechanisms associated with SARS-CoV-2. Other mechanisms of neurological disorders are direct invasion of the brain via the olfactory bulb, carriage across the blood-brain barrier following viremia or entry through infected leucocytes [9].

In conclusion, we reported the first case of CLOCCs associated with SARS-CoV-2 infection. Serological blood tests could be helpful for the diagnosis of SARS-CoV-2-associated complications.

Author contributions AM: data acquisition, drafting the manuscript. AE: data acquisition, drafting the manuscript, IM: data acquisition, revising the manuscript. FV: data acquisition, revising the manuscript. FST: data acquisition, revising the manuscript. NS: data acquisition, revising the manuscript. NG: data acquisition, revising the manuscript. JG: data acquisition, drafting the manuscript.

Funding No funding reported.

Availability of data and material Authors have full access to all of the data.

\section{Compliance with ethical standards}

Conflicts of interest The authors declare that they have no conflict of interest.

Ethics approval Not required.

Consent to participate Not required.

Consent for publication Not required.

Code availability Not applicable.

\section{References}

1. Zhu N, Zhang D, Wang W et al (2020) A novel coronavirus from patients with pneumonia in china, 2019. N Engl J Med 382(8):727-733. https://doi.org/10.1056/NEJMoa2001017

2. Mao L, Jin H, Wang M et al (2020) Neurologic manifestations of hospitalized patients with coronavirus disease 2019 in Wuhan, China. JAMA Neurol. https://doi.org/10.1001/jaman eurol.2020.1127 
3. Tetsuka S (2019) Reversible lesion in the splenium of the corpus callosum. Brain Behav 9(11):e01440. https://doi.org/10.1002/ brb3.1440

4. Ruan Q, Yang K, Wang W et al (2020) Clinical predictors of mortality due to COVID-19 based on an analysis of data of 150 patients from Wuhan, China. Intensive Care Med 46:846-848. https://doi.org/10.1007/s00134-020-05991-x

5. Sethuraman N, Jeremiah SS, Ryo A (2020) Interpreting diagnostic tests for SARS-CoV-2. JAMA. https://doi.org/10.1001/ jama.2020.8259

6. Toscano G, Palmerini F, Ravaglia S et al (2020) Guillain-Barré Syndrome associated with SARS-CoV-2. N Engl J Med. https:// doi.org/10.1056/NEJMc2009191
7. Gutiérrez-Ortiz C, Méndez A, Rodrigo-Rey S et al (2020) Miller Fisher Syndrome and polyneuritis cranialis in COVID-19. Neurology. https://doi.org/10.1212/WNL.0000000000009619

8. Zanin L, Saraceno G, Panciani PP et al (2020) SARS-CoV-2 can induce brain and spine demyelinating lesions. Acta Neurochir (Wien). https://doi.org/10.1007/s00701-020-04374-X

9. Ellul MA, Benjamin L, Singh B et al (2020) Neurological associations of COVID-19 [published online ahead of print, $2020 \mathrm{Jul} 2$ ]. Lancet Neurol. https://doi.org/10.1016/S1474-4422(20)30221-0 